\title{
Optimization of Universitas Riau Data Network Management Using Software Defined Network $(\mathrm{SDN})$
}

\author{
Ery Safrianti* \\ Department of Electrical Engineering, \\ Faculty of Engineering, Universitas \\ Riau, Indonesia
}

\author{
Linna Oktaviana Sari \\ Department of Electrical Engineering, \\ Faculty of Engineering, Universitas \\ Riau, Indonesia
}

\author{
Rian Arighi Mahan \\ Department of Electrical Engineering, \\ Faculty of Engineering, Universitas \\ Riau, Indonesia
}

*corresponding author: Ery Safrianti,esafrianti@eng.unri.ac.id

\begin{abstract}
Computer networks are one of the main parts in the telecommunications system. To support reliable network technology, a centralized network is needed so that network traffic can be managed more easily. Software-Defined Network (SDN) technology is a centralized network that provides a separation between control planes and data planes in different systems. This study discusses the optimization of network management at the University of Riau (UNRI) using SDN. Optimization is done by designing a UNRI computer network in the form of SDN then simulated using the Mininet. Quality of Service $(\mathrm{QoS})$ analysis is performed from the measurement results using Wireshark. The network simulation results give a delay value of $0.506 \mathrm{~ms}, 0 \%$ packet loss, the throughput of $590,392 \mathrm{Mb} / \mathrm{s}$ and jitter of $0.093 \mathrm{~ms}$. The SDN network provides better delay and jitter performance compared to conventional UNRI networks with a delay value of $13,874 \mathrm{~ms}, 0 \%$ packet loss, 635.1 Mb/s throughput and $2.6 \mathrm{~ms}$ jitter. UNRI's SDN network design is worth considering because it has better QoS values, delay, and jitter below ITU standards and conventional networks.
\end{abstract}

Keywords-optimization, computer networks, SDN, UNRI.

\section{INTRODUCTION}

The concept of conventional networks places the data plane and control plane on a device called a router. If a router has been configured using a protocol, it will be very difficult to modify the protocol because the decision to send data is done in private. This problem can be overcome by designing a Software Define Network (SDH) based network. The SDH concept places the data plane and control plane separately. Communication between the data plane and the control plane uses a protocol called Open Flow.

At present, the University of Riau (UNRI) network uses the concept of a conventional network with 4 routers. The router consists of 1 main router and 3 routers underneath. UNRI network management is carried out by 4 people, namely 2 people as admin and 2 people as helpers (support). It also requires 1 supervisor in each faculty to check connectivity with the main router. The different types of routers on the UNRI network cause the need to reconfigure each router if damage occurs. Repair takes $7 \times 24$ hours for the network to work again.

The SDN network provides a solution to optimize UNRI's network management so that it can be done centrally making it easier for admins to manage and repair damage when it occurs on a large scale. SDN also makes it easy to enlarge the UNRI network with the ability to change network behavior in the future.

This research will analyze the Application of Software Defined Network (SDN) in Riau University network which is simulated with MiniNet software. MiniNet is a program to create realistic virtual networks in one machine (virtual machine or cloud) with one command. MiniNet is also software that can be used for development and experimentation with OpenFlow and SDN. The use of MiniNet software is to simulate network design so there is no need for direct testing. This simulation process is carried out to determine the value of Quality of Service (QoS) where the QoS includes: Throughput, Delay, Jitter and Packet Loss.

\section{LITERATURE REVIEW}

\section{A. Introduction of SDN}

Software Defined Network (SDN) is a term that refers to new concepts/paradigms in designing, managing and implementing networks, primarily to support the needs and innovations in this field which are increasingly complex. The basic concept of SDN is to do explicit separation between control and forwarding plane, and then to do abstraction of the system and isolate the complexity of the components or subsystems by defining a standard interface.

SDN architecture can be seen in Figure 1 which consists of 3 layers/fields:

a) Infrastructure (data-plane / infrastructure layer): consists of network elements that can manage the SDN Datapath following the instructions given through the ControlData-Plane Interface (CDPI). 
b) The control plane (control plane/layer): the control entity (SDN Controller) translates application needs with infrastructure by providing appropriate instructions for SDN Datapath and providing relevant information needed by the SDN Application.

c) Application (application plane/layer): located at the top layer, communicating with the system via the Northbound Interface (NBI) [1]

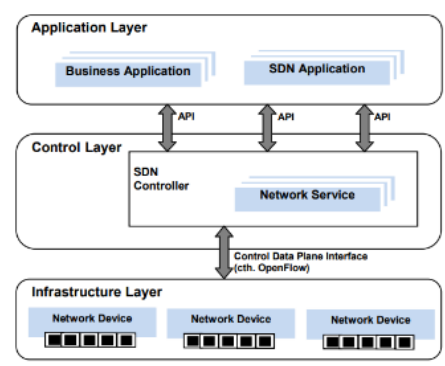

Figure 1: SDN network architecture [2]

\section{B. Software Defined Networks Operations}

SDN operations can be seen in Figure 2. In the figure, SDN networks have 3 divisions, namely SDN devices, controllers and applications. A quick way to understand SDN operations can be seen from below, starting from SDN devices. As shown in Figure 2, SDN devices have a directing function for each data that arrives. SDN devices guide incoming data in which data is represented as flow by the controller. Flow is described by a set of packets sent from one endpoint to another endpoint which is usually defined as IP Address TCP-UDP ports, VLAN endpoints, input ports, and others.

\section{SDN Devices}

SDN devices consist of API (Application Programmer Interface) communication, abstraction layer, and packetprocessing functions, as in Figure 3. In the case of virtual switches, the packet processing function is an embodiment of the hardware for processing logic. Packet (packet-processing logic), seen in Figure 4.

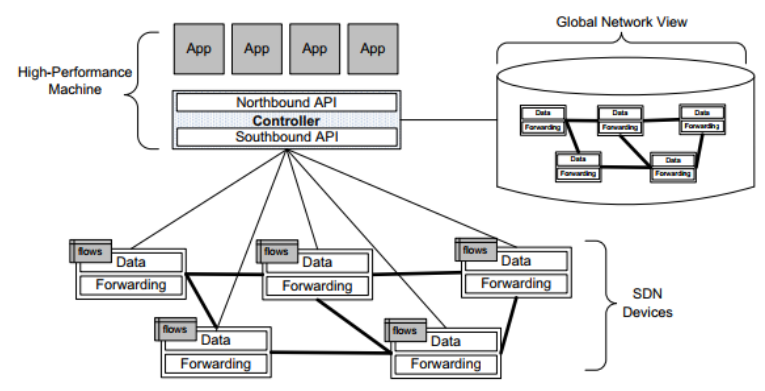

Figure 2: Operation of SDN [3]

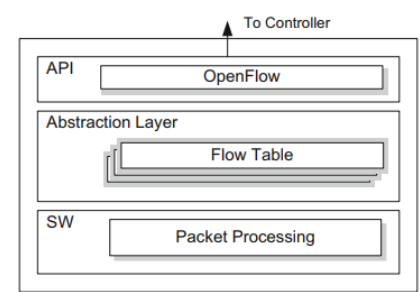

Figure 3: SDN Software Switch Anatomy [3]

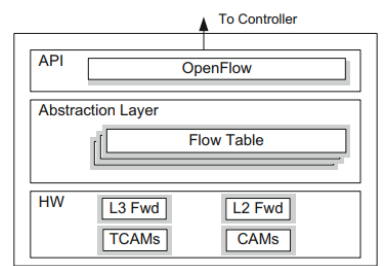

Figure 4: SDN Hardware Switch Anatomy [3]

\section{SDN Controller}

The SDN Controller maintains the shape of the entire network, provides decision policies, and controls all SDN devices consisting of network infrastructure and provides a northbound API for applications. The controller implements decision policies regarding routing, forwarding, directing, load balancing referring to the controller and applications that govern the controller. This can be seen in Figure 5.

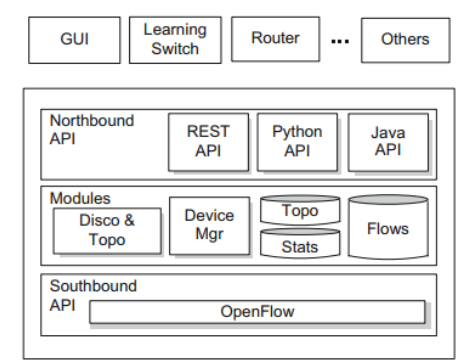

Figure 5: SDN Controller Anatomy [3]

\section{E. SDN Applications}

SDN Applications run on top of the SDN controller, face to face to the network using the northbound API controller. SDN applications are very responsible for managing flow entries programmed on network devices using the API controller to manage the flow. Through the API, these applications can do:

a) Configure packet flow routes through the best path from 2 endpoints.

b) Balance the traffic load through various channels to determine endpoints.

c) React when there is a change in network topologies such as failed links and the addition of devices or paths.

d) Divert traffic with the aim of inspection, authentication, segregation, and security. 


\section{METHODOLOGY}

\section{A. Network Model Planning}

The planning network model that will be used is Riau University network modeling. The network includes all faculties, libraries, and rectorates connected to the University of Riau network. In total there are 9 faculties connected to the UR network, 1 UR library, 1 rectorate, and 1 LPPM (Institute for Research and Community Service). SDN network planning that will be simulated using UR networks using all the nodes mentioned above.

Table 1: Device Configuration on SDN UR network

\begin{tabular}{|c|c|c|}
\hline Device & Type & Version \\
\hline $\mathrm{c} 1$ & $\begin{array}{c}\text { Openflow } \\
\text { Reference }\end{array}$ & - \\
\hline s1-s4 & $\begin{array}{c}\text { Openflow } \\
\text { vSwitch }\end{array}$ & $\begin{array}{c}\text { Openflow } \\
\text { vSwitch 1.0 }\end{array}$ \\
\hline s5 & Legacy Switch & - \\
\hline MONITOR - FK & Host & - \\
\hline
\end{tabular}

Table 1 is the configuration of devices that exist on the designed UNRI SDN network. C1 is a controller of the SDN network by using the Open flow Reference controller type or using an Open Flow type controller. S1-S4 is an Open Flow switch which is connected to the controller so that it has a collaboration with routers on conventional networks. S5 is a type of legacy switch or switch commonly used to share 1 interface to many nodes on the network. Apart from the above is a host who will interact with each other on the network. Figure 7 shows that UR network has 4 routers, on SDN UR network it is replaced by 4 Open Flow switches, each of which is connected to 1 controller. To check the link connected to the network, you can use the pingall command on the CLI controller so that it will look like in Figure 8.

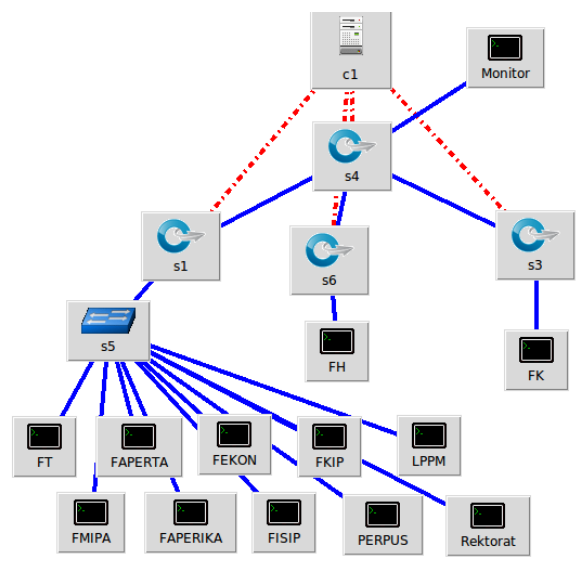

Figure 7: UNRI SDN network in the Mininet software

\section{RESUlt AND DisCUSSION}

\section{A. Delay}

To be able to measure delay, it is necessary to exchange data on the network. In order to flood a network with data, another software called iperf is used. Iperf allows us to flood a network with data and find out the quality of that network. Because the network quality information provided is not as complete as using Wireshark, then using 2 software can display the results of the required QoS information.

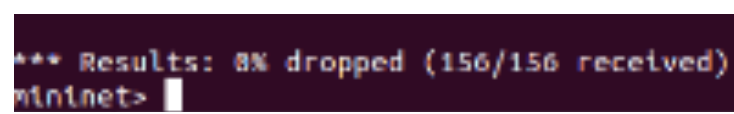

Figure 8: CLI display to check links on the network

In Figure 8 you can see that all links that have been designed are connected, this can be seen in the picture that all packets sent on each link are received with $0 \%$ data being dropped.

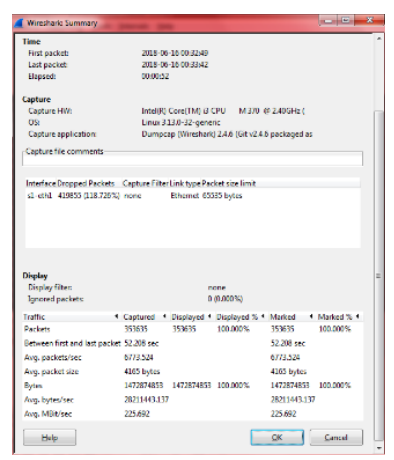

Figure 9: Display of measurement results on Wireshark

Figure 9 is a summary display sub-menu on Wireshark. These sub-menus can be accessed after running a simulation and measuring data passing through a network. The results above are the results of SDN network measurements to find network delays. Measurement of delay on SDN UR networks to see the ability of SDN-based UR networks and compared with conventional UR network delay. Table 2. shows the delay of the UR network.

Table 2: UR Network delay

\begin{tabular}{|c|c|c|}
\hline ITU Standard & Conventional Network & $\begin{array}{c}\text { SDN } \\
\text { Network }\end{array}$ \\
\hline$<150 \mathrm{~ms}$ & $13.874 \mathrm{~ms}$ & $0.506 \mathrm{~ms}$ \\
\hline
\end{tabular}

The table above shows that the value of conventional networks is $13,874 \mathrm{~ms}$. SDN network delay value has a value of $0.506 \mathrm{~ms}$ which has a value of $27 \mathrm{x}$ from the conventional UR network. When referring to ITU standards, both UR networks have very good quality but the SDN network delay value is much better.

\section{B. Packet Loss}

The value of UNRI SDN network packet loss is divided into 3 pieces. Table 3 displays the packet loss results from the UNRI SDN network.

The table above shows the value of packet loss in 3 parts of the UNRI SDN network simulation. Next in table 4 shows the total results of the UNRI SDN network packet loss value 
compared to conventional UNRI networks and the ITU-T G.1010 standard.

Table 3: UNRI SDN network packet loss values

\begin{tabular}{|l|c|c|c|c|}
\hline & Switch 1 & Switch 2 & Switch 3 & Total \\
\hline $\begin{array}{l}\text { Packet Loss } \\
(\%)\end{array}$ & 0 & 0 & 0 & 0 \\
\hline
\end{tabular}

Table 4: Comparison of UNRI network packet

\begin{tabular}{|c|c|c|}
\hline $\begin{array}{c}\text { UNRI Conventional } \\
\text { Network }\end{array}$ & $\begin{array}{c}\text { UNRI SDN } \\
\text { Network }\end{array}$ & $\begin{array}{c}\text { ITU-T } \\
\text { G.1010 }\end{array}$ \\
\hline $0 \%$ & $0 \%$ & $1 \%$ \\
\hline
\end{tabular}

In Table 4, both the conventional UNRI network and SDN UNRI network have the same packet loss of $0 \%$. This means that no data packages are discarded at the time of delivery. When compared with the ITU-T G.1010 standard for 2-way video delivery at $1 \%$, making the UNRI network conventionally and in the form of SDN networks has a very good and reliable network. To see a more detailed data simulation results using Wireshark can be seen in the attachment to the packet loss measurement results.

\section{Throughput}

If a network has a large value of data transmission that can be sent, then it is called bandwidth. Throughput is still related to bandwidth because the value of throughput cannot be more than the value of bandwidth. This is because throughput is a large data transmission value measured in real-time. There is no large standard value of throughput on a network. A good network indicator is the amount of throughput value that is reached and close to the value of the existing bandwidth. The value of throughput can be measured using Wireshark. Throughput values that have been obtained using Wireshark can be seen in the following table 5 .

Table 5: UNRI SDN network throughput

\begin{tabular}{|l|c|c|c|c|}
\hline & Switch 1 & Switch 2 & Switch 3 & Total \\
\hline $\begin{array}{l}\text { Throughput } \\
\text { (Mbit/sec) }\end{array}$ & 523.5 & 46.93 & 19.963 & 590.392 \\
\hline
\end{tabular}

In table 6 it can be seen that the value of throughput simulation results using mininet approaches the bandwidth value on the network. To be able to see the performance of the conventional UNRI network and the UNRI SDN network, table 6 gives the values of both UNRI network throughput.

Table 6: Comparison of UNRI network

\begin{tabular}{|c|c|}
\hline $\begin{array}{c}\text { UNRI Conventional } \\
\text { Network }\end{array}$ & UNRI SDN Network \\
\hline $635.1 \mathrm{Mbit} / \mathrm{s}$ & $590.392 \mathrm{Mbit} / \mathrm{s}$ \\
\hline
\end{tabular}

\section{Jitter (Delay Variation)}

Jitter (Delay Variation) is the value of the variation of the delay that occurs on the network. The smaller the value of jitter, then the network connection formed has good quality. The standard for jitter value refers to ITU-T G.1010 with a large jitter value for video networks that is $<1 \mathrm{~ms}$. Jitter on mininet simulation can be done using iperf. In the iperf command, we add the -u command, which means sending UDP data and measuring the throughput and jitter on the existing link. The results of jitter measurements for the UNRI SDN network can be seen in Table 7 .

Table 7: UNRI SDN network jitter values

\begin{tabular}{|l|c|c|c|c|}
\hline & Switch 1 & Switch 2 & Switch 3 & Total \\
\hline Jitter (ms) & 0.109 & 0.042 & 0.129 & 0.093 \\
\hline
\end{tabular}

In Table 7, the value of jitter on the UNRI SDN network has a very good value which is under $1 \mathrm{~ms}$. To see the comparison value of UNRI network jitter with SDN and existing conventional UNRI network can be seen in Table 8 .

Table 8: Comparison of UNRI network

\begin{tabular}{|c|c|c|c|}
\hline $\begin{array}{c}\text { UNRI Conventional } \\
\text { Network }\end{array}$ & $\begin{array}{c}\text { UNRI SDN } \\
\text { Network }\end{array}$ & $\begin{array}{c}\text { ITU-T } \\
\text { G.1010 }\end{array}$ \\
\hline $2.6 \mathrm{~ms}$ & & 0.093 & $<1 \mathrm{~ms}$ \\
\hline
\end{tabular}

In Table 8, the value of UNRI network jitter with SDN has a better value than conventional networks. Jitter on conventional UNRI networks is of greater value when referring to the ITU standard for sending a 2-way video. However, the jitter value is still below $10 \mathrm{~ms}$ when referring to the Cisco standard for data transmission so that the network can be relied upon (reliable). To see a more detailed data simulation results using iperf can be seen in the attachment to the jitter measurement results.

\section{CONCLUSION}

a) Configuration on UNRI SDN network is done centrally with network automation and virtualization so that the configuration of network devices can be done in 1 day. The time required is shorter compared to conventional networks which require 7 days to configure all network devices.

b) The simulation results of SDN UNRI network delay show a very good value that is $0.506 \mathrm{~ms}$. When compared with the conventional UNRI network, the conventional UNRI network delay value is higher with a value of $13,874 \mathrm{~ms}$.

c) The results of the simulation of packet loss UNRI SDN network showed a very good value that is $0 \%$ of all data that has been sent. This packet loss value is the same as the packet loss value measured on the current UNRI conventional network so that both networks provide a good packet loss value. In the ITU-T G.1010 standard, the tolerance value of packet loss on the network is when sending 2-way video is $1 \%$ and this is the smallest packet loss standard. Both UNRI networks are able to transmit this data.

d) The simulation results of UNRI SDN network throughput shows a good value of $590,392 \mathrm{Mb} / \mathrm{s}$ from $1 \mathrm{~Gb} / \mathrm{s}$ provided. While the value of 
throughput on conventional UNRI networks has a value of $635.1 \mathrm{Mb} / \mathrm{s}$.

e) The results of the UNRI SDN network jitter simulation show very good values when compared to conventional networks. When referring to the ITU-T G.1010 standard, the UNRI SDN network has very good quality with a value below $1 \mathrm{~ms}$ which is 0.093 ms. While jitter on conventional UNRI networks has a value of $2.6 \mathrm{~ms}$

\section{REFERENCES}

[1] Mulyana, Eueung, "Buku Komunitas SDN - RG", Published with GitBook, 2015.

[2] Azwir, M, "Konsep dan Framework Jaringan yang Centralized, Flexible dan Programmable", SDN. Software Defined Networking, 2015.

[3] Goransson, P., Black, C. and Culver, T, "Software defined networks: a comprehensive approach 2nd Edition", Morgan Kaufmann, 2014.

[4] Forouzan, Behrouz A, "Data Communications and Networking 4th Edition", Higher Education, 2017.

[5] ITU-T, "Recommendation ITU-T G.114: One - Way Transmission Time", 2003. 